\title{
Effect of control measures on the pattern of COVID-19 Epidemics in Japan
}

\author{
Tomokazu Konishi ${ }^{\text {Corresp. } 1}$ \\ ${ }^{1}$ Graduate School of Bioresource Sciences, Akita Prefectural University, Akita, Japan \\ Corresponding Author: Tomokazu Konishi \\ Email address: konishi@akita-pu.ac.jp
}

Background. COVID-19 has spread worldwide since its emergence in 2019. In contrast to many other countries with epidemics, Japan differed in that it avoided lockdowns and instead asked people for selfcontrol. A travel campaign was conducted with a sizable budget, but the number of PCR tests was severely limited. These choices may have influenced the course of the epidemic.

Methods. The increase or decrease in the classes of SARS-CoV-2 variants was estimated by analyzing the published sequences with an objective multivariate analysis. This approach observes the samples in multiple directions, digesting complex differences into simpler forms. The results were compared over time with the number of confirmed cases, PCR tests, and overseas visitors. The kinetics of infection were analyzed using the logarithmic growth rate.

Results. The declared states of emergency failed to alter the movement of the growth rate. Three epidemic peaks were caused by domestically mutated variants. In other countries, there are few cases in which multiple variants have peaked. However, due to the relaxation of immigration restrictions, several infective variants have been imported from abroad and are currently competing for expansion, creating the fourth peak. By April 2021, these foreign variants exceeded $80 \%$. The chaotic situation in Japan will continue for some time, in part because no effort has been made to identify asymptomatic carriers, and details of the vaccination program are undecided. 
1 Effect of control measures on the pattern of COVID-19

2 Epidemics in Japan

3 Tomokazu Konishi ${ }^{1}$

$4{ }^{1}$ Graduate School of Bioresource Sciences, Akita Prefectural University, Akita 010-0195, Japan 5

6 Corresponding Author:

7 Tomokazu Konishi ${ }^{1}$

8 Kaidobata Nishi, Shimoshinjyo Nakano, Akita 010-0195, Japan

9 Email address: konishi@akita-pu.ac.jp 


\title{
Effect of control measures on the pattern of COVID-19 Epidemics in Japan
}

\author{
Tomokazu Konishi Corresp. 1
}

${ }^{1}$ Graduate School of Bioresource Sciences, Akita Prefectural University, Akita 010-0195, Japan

Corresponding Author:

Tomokazu Konishi ${ }^{1}$

Kaidobata Nishi, Shimoshinjyo Nakano, Akita 010-0195, Japan

Email address: konishi@akita-pu.ac.jp

\section{Abstract}

Background. COVID-19 has spread worldwide since its emergence in 2019. In contrast to many other countries with epidemics, Japan differed in that it avoided lockdowns and instead asked people for self-control. A travel campaign was conducted with a sizable budget, but the number of PCR tests was severely limited. These choices may have influenced the course of the epidemic.

Methods. The increase or decrease in the classes of SARS-CoV-2 variants was estimated by analyzing the published sequences with an objective multivariate analysis. This approach observes the samples in multiple directions, digesting complex differences into simpler forms. The results were compared over time with the number of confirmed cases, PCR tests, and overseas visitors. The kinetics of infection were analyzed using the logarithmic growth rate. Results. The declared states of emergency failed to alter the movement of the growth rate. Three epidemic peaks were caused by domestically mutated variants. In other countries, there are few cases in which multiple variants have peaked. However, due to the relaxation of immigration restrictions, several infective variants have been imported from abroad and are currently competing for expansion, creating the fourth peak. By April 2021, these foreign variants exceeded $80 \%$. The chaotic situation in Japan will continue for some time, in part because no effort has been made to identify asymptomatic carriers, and details of the vaccination program are undecided.

\section{Introduction}

The worldwide COVID-19 pandemic continues as variants mutate rapidly (Konishi 2021c). In Japan, the epidemic began with the spread from a cruise ship. Hitherto, there have been four peaks. As of June 2021, COVID-19 has not been contained, people living in major cities have been asked to stay at home voluntarily, and travel to Japan from abroad is restricted. 
49 The responses to COVID-19 in Japan were different from those of some countries that succeeded 50 in controlling the disease. Strict lockdowns were not carried out; instead, Japan issued states of 51 emergency three times, urged the public to refrain from going out unnecessarily and eating out 52 late at night, and cancelled events. These measures were merely a request for self-restraint, and 53 there were no confirmations, penalties, or compensation. Schools were closed only during the 54 first state of emergency. In the midst of the second peak, the government launched a travel55 promoting campaign, "Go To travel," which provides residents with subsidies of up to $50 \%$ on 56 transportation, hotels, restaurants, and shopping; 26 billion dollars was proposed for the budget 57 to do so. However, the number of public polymerase chain reaction (PCR) tests is fairly low, and 58 testing is only performed on patients with obvious symptoms. Initially, the plan was to find 59 60 clusters and infection routes, but this scope was beyond the capacity of the investigation agency and has already been substantively abandoned. The number of small vendors performing PCR tests is increasing, but the results are not counted in official records.

Immigration procedures have often been criticized for being loose (Edamatsu et al. 2021). Upon arrival, infection is checked via PCR or antigen testing. Visitors are then asked to stay in one place for two weeks, but this is not compulsory, and 20\% became uncontactable.

The same laissez-faire policy is applied to domestic PCR-positive patients. There is an insufficient number of facilities in which to isolate mild and asymptomatic patients. As of May 15, 2306 people in Tokyo (Tokyo Metropolitan Government 2021) and 13499 people in Osaka (Osaka Prefecture 2021) were asked to stay at home or in hotels. However, this request for self-

72

73

74

75

76

77

78

79

80

81

82

83

84

85

86

87

88 restraint was not compulsory.

Medical resources have been exhausted. The patients who were staying home (or in a hotel) were not administered medicine or oxygen. Among them, 1,340 people in Tokyo and 2,799 people in Osaka have requested hospitalization, but 122 people have died without being hospitalized as far as the police have acknowledged (Akahata 2021). From January 6-28, 110-90\% of the beds for severely ill patients in Tokyo hospitals were occupied, and on April 21, and 80\% of such beds were occupied in Osaka hospitals (NHK 2021). At the end of April, of the 13 calls for emergency services, eight were turned down in an emergency hospital in Osaka, which is a highly unusual situation (Inoue \& Tanabe 2021). In this study, we examined the situation of epidemics with respect to changes in the relative proportions of virus variants as well as kinetics.

All sequence data available in GISAID (Elbe \& Buckland-Merrett 2017) were observed in an objective manner by considering the sequence matrix as a multivariate variable and applying principal component analysis (PCA; Jolliffe 2002). This approach is different from phylogenetic trees (Yang \& Rannala 2012), which require many unverifiable assumptions that reduce the objectivity of the analysis (Ellis \& Silk 2014). Because several directions of differences can be observed individually, fine classification can be performed with high reproducibility. These data 
89

90

91

92

93

94

95

96

97

98

99

100

101

102

103

104

105

106

107

108

109

110

111

112

113

114

115

116

117

118

119

120

121

122

123

124

125

126

were observed in chronological order and compared with the number of confirmed cases, the number of overseas visitors, and the number of PCR tests performed. It should be noted that a tree does not produce data in a format that can be compared to other information. Therefore, it is difficult to integrate time-course studies with a phylogenetic approach. Additionally, a kinetic approach was applied using the logarithmic growth rate.

\section{Materials \& Methods}

Data source. Nucleotide sequences of 15,746 samples in Japan were obtained from GISAID (Elbe \& Buckland-Merrett 2017) on May 5, 2021. Sequences were aligned using DECIPHER (Wright, 2015), which uses secondary structure predictions in the process. Because PCA is not sensitive to differences caused by alignment methods or to the parameter conditions of calculations (Konishi et al. 2019), DECIPHER was selected for its faster calculations with a large number of sequences. The aligned sequences were summarized by PCA, as explained in the next section, and then the PCs were compared with the collection date of the sample. All calculations were performed using R (R Core Team 2020). The ID, acknowledgements, and scaled PC (sPC) of the samples and the sPC of the bases are available from Figshare (Konishi 2021b). The number of confirmed cases and the number of PCR tests were obtained from the Ministry of Health, Labour and Welfare, Japan (Ministry of Health, Labour and Welfare Japan 2021). Those of Tokyo were from the home page of Tokyo City (Tokyo Metropolitan Government 2021). The number of foreign visitors was obtained from an official statistics counter (e-Stat 2021). The number of current cases and PCR tests in the other countries were obtained from the official homepage of the Ministry of Health of the corresponding countries.

The nomenclatures of the variants are based on the Pango Lineage in GISAID, version 2021-0527 (Rambaut et al. 2020).

$P C A$. This analysis represents the differences among samples of multivariate data through a set of common directions, which are shown as independent vectors (Jolliffe 2002). Here, the samples were mutations that evolved from the original virus. Hence, the samples will fall into several related groups; each group is different from the others, with a unique direction common to the group. The sequence matrix is converted to a stack of Boolean vectors to allow for calculation, replacing specific positions from 0 to 1 for the corresponding bases (Konishi et al. 2019). When $m$ is the number of samples (in reality, to cover sequences with $n$ bases, the length of a Boolean vector becomes $5 n$, i.e., A, T, G, C, and (-); here, for simplicity, it is described as below), the matrix $X$ is given as follows:

$X=\left(\begin{array}{ccc}01_{11} & \cdots & 01_{1 n} \\ \vdots & \ddots & \vdots \\ 01_{m 1} & \cdots & 01_{m n}\end{array}\right)$.

Next, the average of the samples, $a$, is found, and $X$ is centered by subtracting the average from each row: $a=\left(a_{1}, a_{2}, \cdots a_{n}\right), C=X-a$. This centered matrix, $C$, was applied to the PCA. It is 
127

128

129

130

131

132

133

134

135

136

137

138

139

140

141

142

143

144

145

146

147

148

149

150

151

152

153

154

155

156

157

158

159

160

161

162

163

164

165

166

subjected to singular value decomposition, $C=U \Sigma V^{*}$, where $U$ and $V$ are unitary matrices that specify the directions of the differences. According to the definition of the unitary matrix, $V^{*}$ $V=I$ and $V V^{*}=I$, the scale of each column and row is one, $V^{*} V=I$, and $V V^{*}=I$. Each of their columns can be regarded as vectors that convert $C$ to be represented on a specific axis. $\Sigma$ is a diagonal matrix that records the scaling of each axis in descending order.

The PCs for the samples, $S$, were found to be $S=C V=U \Sigma$. The $C V$ indicates the rotation of $C$ around the central origin, retaining the shape of $C$. The results of the rotation are along the axes. This is the same as $U \Sigma$, which is a unitary matrix given the scale. Each of the columns of $S$ represents the PCs: the leftmost column is PC1, and the second column is PC2. The descending character of $\Sigma$ orders the scaling of PCs. All of the information is conserved, and all calculation steps are reversible. Once found, axis $V$ can be applied to other sets of matrices (Konishi 2015). This characteristic is beneficial when applying classification to newly found samples. Here, the axes used were found by using worldwide samples.

PCs for bases, $B$, can be found in the diagonal direction of the above, $C^{*}=V \Sigma U^{*}$, as $B=C^{*}$ $U=V \Sigma$. Therefore, $S$ and $B$ are inextricably linked; for example, samples with many positive $B$ in an axis will become highly positive in $S$ along the same axis. In contrast, a sample's characteristics that show a high score on an axis will appear on the same axis as $B$. This characteristic is beneficial for identifying new variants and their corresponding mutations. A mutation(s) characteristic of a group of samples gives a particular $S$ value along an axis. The mutation(s) appear in $B$.

To enable comparisons with datasets with different sizes of $n$ or $m$, PCs can be scaled to different sizes (Konishi 2015). The scaled versions of $S$ and $B, \mathrm{sPC}$ for samples and sPC for bases, are $S /$ $\sqrt{n}$ and $B / \sqrt{m}$, respectively. These scaled versions were used in this study.

For these averages $a$ and axes $V$, we used a sequence matrix collected evenly from all over the world as of October 2020 (Konishi 2021c) to allow for comparison of the variants that are prevalent around the world with those prevalent in Japan. The axes represent the changes that have occurred in some countries, including Japan. If a change occurred in Japan, a specific value would appear on the same axis. In addition, to observe newer variants, a new set of axes at May 2021 was also used. Both sets of axes can be downloaded from Figshare (Konishi 2021b).

The conditions for finding variants that produced peaks in each country were as follows (Konishi 2021a):

\section{(original axes)}

group 0: $-0.004<\mathrm{PC} 1<0.004$, PC2 $<0.004$

B.1.177: PC62 $>0.0035$, PC63 $>0.009$ 
167

168

169

170

171

172

173

174

175

176

177

178

179

180

181

182

183

184

185

186

187

188

189

190

191

192

193

194

195

196

197

198

199

200

201

202

203

204

group 2: $\mathrm{PC} 2>0.004$

B.1.160: $\mathrm{PC} 118>0.002$

B.1.2: $\mathrm{PC} 28>0.01$

(new axes)

B.1.1.7: $\mathrm{PC} 1<-0.016$

B.1.351: $\mathrm{PC} 23>0.006 \& \mathrm{PC} 25>0.006$

B.1.617.1: PC56 $>0.004 \&$ PC60 $>0.001$

B. 1 : $\mathrm{PC} 21>0.0024$

The variants that arose in Japan were found with the help of k-means (Hartigan \& Wong 1979), which is a non-hierarchical method that classifies samples into a given number of groups. Samples were divided into eight groups, and for each group, each PC axis was compared to the collection date. The number of groups, eight, has an arbitrary nature. It was chosen since it was the largest number, after repeating this process several times, that did not produce small groups that were almost worthless. Although k-means is a method with low reproducibility, we selected the trials where the grouping did not differ greatly from other trials as a result of the repetition. This arbitrariness is an inevitable consequence of the classifications as it is arbitrary which mutations are considered as independent variants. Samples that showed a specific value in an axis (or multiple axes) at a specific time period were taken as new variants, and the PC value was used to define it (rather than the results of k-means). This process was repeated several times, including by using the new axes, and a total of 10 variants were identified. Each variant was compared to the variant that produced the first peak (shown in tangerine) to determine which amino acids had changed (Table 1).

\section{Kinetics}

When the infection is early and there are many uninfected people, we can use a simple model (Wallinga \& Lipsitch 2007) in which the number of confirmed cases on the $n$th day, $c_{n}$, increases or decreases exponentially. Hence, the logarithmic growth rate $K$ was calculated to observe the fluctuation of cases in more detail. When a variant grows exponentially, $K$ becomes constant, and $c_{n}$ increases with constant doubling time, $d=1 / K, c_{n}=c_{0} 2^{n / d}=c_{0} 2^{K n}$.

Taking the logarithm of both sides gives the following formula: $\log _{2} c_{n}=K n+\log _{2} c_{0}$.

Differentiating these two sides by $n$, we find $K$ as $\operatorname{dlog}_{2} c_{n} / \mathrm{d} n=K$.

Hence, changes in $K$ at any $n$ were estimated as $K_{n}=\left(\log _{2}\left(c_{n+7} / c_{n}\right)\right) / 7$; the seven-day difference was set to minimize the fluctuations due to the day of the week. Because the base was set to 2 here, the inverse of $K$ shows the doubling time; for example, if $K$ is 0.1 , the doubling time is 10 days. 
205 For convenience, we also included the more popular basic reproduction number $R_{0}$. Here, the 206 infection period of $\tau$ is assumed to be constant at five days (Alene et al. 2021). Eq.1 can be 207 transformed using $R_{0}$ and $\tau$ by approximating the event based on the idea that an average carrier 208 infects $R_{0}$ people on day $\tau$ of infection:

$209 c_{n}=c_{0} R_{0}^{n / \tau}$

210 From Eqs. 1 and 2, we get $R_{0}=2^{K \tau}$.

211

212

213

214

215

216

217

218

219

220

221

222

223

224

225

226

227

228

229

230

231

232

233

234

235

236

237

238

239

240

241

242

243

Because of sampling errors, the estimations of $c_{n}, K$, and $R_{0}$ fluctuated; they were smoothed by a locally weighted scatter plot smooth function, which finds the center of data by using moving weights and robust calculations (Cleveland 1979).

Additionally, as $K$ changed linearly over time, the linear relationship was approximated by robust line fitting (Tukey, 1977). Therefore, the slopes of the lines indicate the changes per day.

\section{Results}

With the first and second axes of the original PCA, the variants thus far were divided into groups of 0 to 3 (Fig. 1A). These groups were commonly observed in other countries on all continents (Konishi 2021c).

Fig. 1B shows how the rates of these groups changed (left axis). The green and pink backgrounds represent the period of the state of emergency and the Go To travel campaign, respectively. The same panel also shows the number of detected cases, PCR tests, and foreign visitors (right axis). The number of public PCR tests, which were given only to those with clear symptoms, was 19 times the number of confirmed cases. This testing rate is lower than that of some successful countries by one to two orders of magnitude; for comparison, on Feb 22, 2021, Australia has 39 estimated cases and 34,800 PCR tests per day (Department of Health, Australia 2021). For the last 30 days from the same date, New Zealand had 802 positive results and 194,233 negatives (Ministry of Health, New Zealand 2021). Singapore had 196 positives while performing 32,100 PCR tests per day (Ministry of Health, Singapore 2021). Iceland had only one domestic infection within seven days but still performed one thousand tests per day (Iceland Government 2021).

Until March 2020, only Group 3 variants were identified. This group includes the earliest variants reported from China (Wu et al. 2020; Zhang et al. 2018) and those found on the cruise ship Diamond Princess. Since then, this group has disappeared worldwide, perhaps because of lower infectivity relative to other groups (Konishi 2021c).

Subsequently, Group 1 was the mainstream group throughout 2020 (Fig. 1B). It peaked in April 2020 and was eventually replaced by more mutated variants; Fig. 2A-C show typical examples of when and how many such variants existed. It should be noted that what defines a variant is 
244 arbitrary. In fact, the classification here sometimes disagrees with the Pango lineage (Rambaut et 245 al. 2020), which is based on phylogenetic tree analysis. For instance, most of the domestic 246 variants were categorized as B.1.1.214, with the exception of the latest blue-green variant that 247 was categorized as R.1. However, as Fig. 2 shows, these variants are objectively distinguished 248 from others in that they have their own characteristic PC and mutations (Table 1). Here, a new 249 variant was defined as one with a unique value in the PC axis and that appeared during a specific 250 time period. The same characteristics were also observed for the variants that peaked in each 251 country. A clear example is variant B.1.1.7, which once occupied England. Because it has many 252 mutations, it showed PC values that were significantly different from those of the rest of the 253 population (Fig. 2D).

254

The number of people entering Japan was on the rise, with an average of approximately 130 thousand people per day arriving in Japan in 2019. The first state of emergency reduced this number to less than one thousand (Fig. 1B). However, the number of visitors has gradually increased since then. Visitors were asked to voluntarily quarantine in hotels for two weeks; however, as already mentioned, the inspection and quarantine of entry were not strictly enforced. Accordingly, as the number of visitors increased, overseas variants appeared in Japanese cities.

261

262 Since November 2020, variants that created the peak overseas have also appeared in Japan (Fig. 3). Variant B.1.2 was, the variant that long swept the USA and even peaked in Australia. Variant B.1.177 swept Europe. Variant B.1.160 (Fig. 3C), which was more prevalent in France during the same period, was also observed. Variants B.1.525 and B.1.1.317, which appeared in Europe, were also found, although it accounted for only in a small percentage of cases. Variant B.1.1.207, which was prevalent in North America, was also confirmed. In March, B.1.351 and P.1, which were endemic in Africa and South America, respectively, were confirmed; these should continue to be closely observed. However, in February, B.1.1.7, a variant from England, increased rapidly and became the predominant variant (Fig. 3D). At the end of April, B.1.617.1, 272 which was the predominant variant in India, appeared and spread rapidly.

In Fig 4A, we can see the response of the government to changes in the number of variants in more detail. Here, the logarithmic growth rate $K$ of the confirmed cases is shown to make it easier to understand the triggers for the increase and decrease in the number of patients. A more widely used basic reproduction number, $R_{0}$, is also shown (Fig. 4C); however, the calculation of this number is less objective, and the value may not represent the true $R_{0}$ because it requires the selection of an infection model and the estimation of the average duration of infection (Delamater et al. 2019).

280

If the cases grow exponentially, $K$ will show a constant positive value; however, the value increased and decreased linearly (Fig. 4A). The movement is approximated by the light blue and orange dashed straight lines. The accompanying number is the slope, indicating the increase or 
284 decrease in $K$ per day. The linear relationships were stable for several weeks, and after a short 285 transition period, they moved on to the next linear phase. $K$ increased in the valleys between the 286 peaks of the confirmed cases. In addition, $K$ decreases in the top half of a peak. The peak apex 287 coincides with the time when $K=0$ during the descent of $K$. There is a delay of one to two 288 months from the time when $K$ begins to rise or fall until there is a clear change in the number of 289 confirmed cases. $K$ is the determinant for the number of cases; the more positive days in $K$, the 290 more rapid the increase in confirmed cases will be.

291

292

293

294

295

296

297

298

299

300

301

302

303

304

305

306

307

308

309

310

311

312

313

314

315

316

317

318

319

320

321

322

323
In the first peak in April 2020, the first state of emergency was declared, but $K$ was already in the middle of a rapid decline. The variant that caused the peak, shown in tangerine, had characteristics only as of the group 1 variant; the variant disappeared immediately. Immediately after the peak, the light blue variant began to increase, and $K$ bottomed out and began to rise. However, the state of emergency was discontinued in the midst of the rising $K$.

Even when the light blue variant increased to form the second peak, a state of emergency was not declared; instead, a "Go To travel" campaign was launched instead (Fig. 4A). Despite this incoherent policy, the increase in light blue variants subsided (September 2020), but the epidemic did not stop. At least nine new variants emerged (Fig. 4A and Table1), and their proliferation jointly kept $K$ positive, producing a sharp increase in the number of confirmed cases (Fig. 4A). The cases continued to grow during the campaign (Fig. 4A, December 2020), but $K$ began to decrease spontaneously (Fig. 4B). It should be noted that even with the highly infectious B.1.1.7 variant, $K$ decreased during this period (Fig. 4A, dotted line). Eventually, the campaign was suspended, and the second state of emergency was issued.

During the second state of emergency, $K$ began to rise again. This rise was due to the fact that two new variants began to proliferate. The blue-green variant R.1 is one of the newest domestic variants and has two mutations in its spike; another blue variant was identified as B.1.1.7 (Fig. 4A, Table 1). As a natural consequence, after the state of emergency was discontinued, $K$ continued to increase and reached a peak, and the number of infected people increased again. The third state of emergency was not issued until $K$ decreased again, and the number of infected people continued to increase. B.1.1.7 showed a higher $K$ and $R_{0}$ than the whole but peaked earlier; this is likely due to the increase in the next variant, B.1.617.1 (Fig. 3D).

Before the Go To Travel campaign, the change in $K$ in Tokyo was about two weeks ahead of that nationwide. However, after the campaign, the difference was almost eliminated (Fig. 4B).

The B.1.1.7 variant has been mutating since it arrived in Japan, similar to the domestic variants (Fig. 5). For a period of time, it looked as if the increase had slowed (Fig. 4A and 5B, dotted line). However, it has begun to increase again as of the writing of this paper, likely because of these mutations. Such mutations have been found along several axes, producing several variants. 
324

325

326

327

328

329

330

331

332

333

334

335

336

337

338

339

340

341

342

343

\section{Discussion}

345 Group 1 variants were changing in Japan in 2020, causing three peaks of the epidemic. At the

346

347

348

349

350

351

352

353

354

355

356

357

358

359

360

361

362

363

364

It should be noted that the linear increase or decrease of $K$ was not affected by the states of emergency (Fig. 4A). Except for the first peak, $K$ decreased at the same rate. For the second peak, a state of emergency was not issued. For the fourth peak, one was issued after the descent of $K$. During the third peak, although the state of emergency seemed to have an effect, the rate of decrease in $K$ was the same as during the other two. This was also true when the data were selected solely for Tokyo, where the states of emergency were issued (Fig. 4B). The rate of increase of $K$ also remained constant regardless of the presence or absence of the states of emergency. If declarations alter the rate of change in $K$, then these lines should be curved.

The inadequacy of the immigration policies not only allowed powerful variants from abroad to enter the country but also led to the transfer of variants. The three major variants that formed the third peak (Fig. 4A, coral red, flesh pink, and green) spread to South America, where they accounted for about $10 \%$ of the epidemic by the end of February 2021 (Fig. S1). In addition, R.1 (Fig. 4A, blue-green) caused approximately 1,000 cases in North America in April 2021. A variant in B.1 (group 0 and new PC21 > 0.0024) appeared in January 2020. As group 0 has been rather minor, Japan may not be its origin country, but it was the only country in which an epidemic was confirmed at that time. This variant became prevalent in the USA from August to September 2020, accounting for a quarter of the total cases. end of the year, the number of variants originating overseas began to rise, leading to a large fourth wave from May to June 2021. PCA was helpful in properly identifying and classifying variants. If we had not been able to distinguish that these were many separate variants, we could not have known why $K$ was positive during the Go To travel campaign or why cases waved, forming peaks. Additionally, the variants that were transferred overseas were buried among the other variants.

Each ascent of $K$ was caused by a new variant (Fig 2, 4A, and 5). Currently, SARS-C0V-2 is considered to be in the process of acclimatization to humans (Konishi 2021c). A variant that has mutated to be more infectious would nullify the countermeasures people have been taking, and $K$ would, therefore, increase. The change did not necessarily occur in the spike protein (Table 1), which would be the target of immunity (Harvey et al. 2021). This shows that herd immunity has not yet been established and may be the reason why B.1.351, which has many mutations in the spike, has not caused a large epidemic. Unfortunately, the increased number of patients has increased the possibility of further mutations, creating a vicious cycle.

There is a marked difference in government measures between countries that succeeded in controlling the virus and those that did not. To maintain cleanliness, it is necessary to identify and isolate virus carriers, and a lockdown is essential until this task is completed. A sufficient

Peer] reviewing PDF | (2021:03:58872:3:0:NEW 16 Aug 2021) 
365

366

367

368

369

370

371

372

373

374

375

376

377

378

379

380

381

382

383

384

385

386

387

388

389

390

391

392

393

394

395

396

397

398

399

400

401

402

403

404

405

406

number of repeated PCR tests are required for this task. Otherwise, we will not be able to control a pathogen that is growing exponentially and mutating rapidly because an epidemic can emerge from a single asymptomatic carrier (Johansson et al. 2021). This is a mathematical truism, but the reality is supporting it. Repeated and incomplete blockades are the fallacy equivalent of using a small piece of force instead of the utmost use of force, which has long been considered the wrong tactic (Clausewitz 1832). This is because such incomplete measures only increase the economic cost without any practical benefit. Vaccination is another promising strategy, but Japan is lagging behind, and the program remains undecided (Ministry of Health Labour and Welfare, Japan 2021).

Japan is unique in that many variants are simultaneously prevalent. In contrast, in many other countries, one major variant, which is mutated domestically or imported from abroad, is prevalent (Konishi 2021c); for example, the epidemic Group 2 variant, B.1.2, may have mutated domestically in the USA and subsequently entered Australia and Japan, causing new epidemics in those countries (Fig. 3). The earliest record of B.1.177, the pan-European variant, was from Spain, which was occupied by its parental variant. This variant has caused epidemics in many European countries. The new variants B.1.351 and B.1.617.1 may have entered South Africa and India from outside. They may have mutated in areas without sequencing, as no possible parental variants have been recorded. They all once accounted for most of the cases in the respective country. The situation in Japan is a result of the fact that the domestic variants were not contained as well as of the importation of highly infectious variants after reckless relaxation of the border. Variant B.1.1.7 is changing (Fig. 5), similar to the domestic variants (Fig. 4A, colored lines). Variant B.167.1 will likely mutate as well, producing a new variant that is responsible for another peak.

To date, three states of emergency have been issued. These specifically recommend working remotely and prohibit eating out at night, especially the serving of alcoholic beverages. However, the number of PCR tests did not increase, and positive patients were not isolated. There are no restrictions on people's activities, and the infamous jam-packed train remains unchanged. Each state of emergency was untimely; they were not issued until $K$ began decreasing spontaneously, and they ended in the midst of the ascent of $K$ (Fig. 4A). In addition, the effect of the states of emergency was almost ineffective; it could not stop the increase of the next variant, and it did not alter the rates of the changes in $K$. It is clear that such half-hearted measures were completely ineffective. It is likely that the infection is spreading through non-meal-related situations, such as daily life at home, school, or work or commuting on trains.

The Go To travel campaign may have spread this virus over a wide area of Japan. Until that point, the timing of the increase and decrease of the value of $K$ in Tokyo had been around two weeks ahead of the rest of the country. It should be considered that the loss of this difference in timing is the result of people mixing together due to increased human flow (Fig. 4B). This may give the virus more chances to cause infection and hence to mutate, and it has expanded the number of confirmed cases during the third peak. The only government measure that has worked

Peer) reviewing PDF | (2021:03:58872:3:0:NEW 16 Aug 2021) 
407 thus far may have been the cancellation of this campaign. It was not the government's declaration 408 that lowered $K$ but, perhaps, the voluntary efforts of the people. Nevertheless, it is also true that 409 the government has repeatedly released the information necessary for the efforts, and this should 410 be commended.

411

412 The epidemic did not increase exponentially, but was even more explosive. The value of $K$ 413 consistently increased or decreased (Fig. 4); such linear movement of $K$ was ubiquitously 414 observed in other countries as well (Fig. S2). The constancy of $K$ is the basis of various 415 mathematical models, but the actual mechanism of infection seems to be different in SARS416 CoV2. The mechanism of the linear increase and decrease of $K$ is currently unknown; it seems 417 that a novel mathematical model will be required to explain this movement. The value of $R_{0}$ also 418 fluctuates; when $K$ changes linearly, $R_{0}$ will do so exponentially; hence, this parameter is 419 unstable and not suitable as an indicator. Additionally, it is difficult to compare $R_{0}$ because of the 420 problem of differences in the models (Delamater et al. 2019). It would be inappropriate to 421 continue using $R_{0}$ as long as the standing mathematical models do not explain the situational 422 reality.

423

The value of $K$ will be useful in predicting infection status, as the ascent or descent precedes the fluctuations in the number of cases by a month or two. This index can be easily calculated. Unlike $R_{0}$, this is a raw physical value, which does not require a complex model or estimation of $\tau$. Accordingly, $K$ would be a better choice when comparing results among different situations, at least at the present time.

429

430

431

\section{Conclusions}

PCA sensitively detected new variants and allowed us to compare them over time. As a result, several aspects were clarified. The original intention of Japan to block the transmission route and resolve the infection was unsuccessful. Moreover, the partial lockdown that requests only selfsuppression failed to contain the virus. Meanwhile, domestic variants continued to mutate and caused multiple epidemic peaks. A poorly managed waterfront operation failed, and a number of highly infectious variants were introduced into the country, while others were taken out of the country. As a result of these inappropriate measures, multiple infectious variants have spread and have taken root in Japan. Over $80 \%$ of the present variants are of foreign origin, and they seem to continue changing. If the borders are relaxed again, more new variants will emerge, and the rooted variants will be released from the country to the world.

445 We would like to thank Editage (www.editage.com) for English language editing. 
447

448

449

450

451

452

453

454

455

456

457

458

459

460

461

462

463

464

465

466

467

468

469

470

471

472

473

474

475

476

477

478

479

480

481

482

483

484

485

486

487

488

489

490

491

492

493

\section{References}

Akahata Shinbun. 2021. 122 people died at home by Corona Infetion. 11 June 2021.

Alene M, Yismaw L, Assemie MA, Ketema DB, Gietaneh W, and Birhan TY. 2021. Serial interval and incubation period of COVID-19: a systematic review and meta-analysis. BMC Infectious Diseases 21:257. 10.1186/s12879-021-05950-x

Clausewitz Cv. 1989 On War. Translated by Howard H in 1989. Princeton Univ Pr.

Cleveland WS. 1979. Robust Locally Weighted Regression and Smoothing Scatterplots. Journal of the American Statistical Association 74:829-836. 10.1080/01621459.1979.10481038

Delamater PL, Street EJ, Leslie TF, Yang YT, and Jacobsen KH. 2019. Complexity of the Basic Reproduction Number $(\mathrm{R}(0))$. Emerging Infectious Diseases 25:1-4. 10.3201/eid2501.171901

Department of Health, Australia. 2021. Coronavirus (COVID-19) current situation and case numbers. Available at https://www.health.gov.au/news/health-alerts/novel-coronavirus2019-ncov-health-alert/coronavirus-covid-19-current-situation-and-case-numbers.

e-Stat. 2021. Immigration statistics. Available at https://www.e-stat.go.jp/

Edamatsu Y, Shimoji K, and Satou T. 2021. 300 people a day are not responding to the waiting list after entering Japan. Asahi Shinbun. Available at https://www.asahi.com/articles/ASP5073J3P50UTFK01C.html

Elbe S, and Buckland-Merrett G. 2017. Data, disease and diplomacy: GISAID's innovative contribution to global health. Glob Chall 1:33-46. 10.1002/gch2.1018

Ellis G, and Silk J. 2014. Scientific method: defend the integrity of physics. Nature 516:321-323. 10.1038/516321a

Harvey WT, Carabelli AM, Jackson B, Gupta RK, Thomson EC, Harrison EM, Ludden C, Reeve R, Rambaut A, Peacock SJ, Robertson DL, and Consortium C-GU. 2021. SARS-CoV-2 variants, spike mutations and immune escape. Nature Reviews Microbiology. 10.1038/s41579-021-00573-0

Iceland Government. 2021. COVID-19 in Iceland - Statistics. Available at https://www.covid.is/data

Inoue S, and Tanabe M. 2021. Osaka: what about hospitals under emergency declarations? 24 hours of close contact "on the verge. NHK. Available at https://www3.nhk.or.jp/news/html/20210528/k10013050031000.html

Johansson MA, Quandelacy TM, Kada S, Prasad PV, Steele M, Brooks JT, Slayton RB, Biggerstaff M, and Butler JC. 2021. SARS-CoV-2 transmission from people without COVID-19 symptoms. JAMA Network Open 4:e2035057-e2035057. 10.1001/jamanetworkopen.2020.35057

Jolliffe IT. 2002. Principal component analysis. New York, NY: Springer-Verlag.

Konishi T. 2015. Principal component analysis for designed experiments. BMC Bioinformatics 16 Suppl 18:S7. 10.1186/1471-2105-16-S18-S7

Konishi T, Matsukuma S, Fuji H, Nakamura D, Satou N, and Okano K. 2019. Principal Component Analysis applied directly to Sequence Matrix. Scientific Reports 9:19297. 10.1038/s41598-019-55253-0

Konishi T. 2021a. Continuous mutation of SARS-CoV-2 during migration via three routes. Figshare. 10.6084/m9.figshare.12365777.v1

Konishi T. 2021b. Pattern of COVID-19 Epidemics in Japan Available at https://figshare.com/authors/Tomokazu_Konishi/

Konishi T. 2021c. Progressing adaptation of SARS-CoV-2 to humans. bioRxiv : Available at https://www.biorxiv.org/content/biorxiv/early/2021/01/15/2020.12.18.413344.full.pdf

Peer] reviewing PDF | (2021:03:58872:3:0:NEW 16 Aug 2021) 
494

495

496

497

498

499

500

501

502

503

504

505

506

507

508

509

510

511

512

513

514

515

516

517

518

519

520

521

522

523

524

525

526

527

528

529

530

531

532

533

534

Ministry of Health Labour and Welfare, Japan. 2021. About new coronavirus infection. Available at https://www.mh/w.go.jp/stf/seisakunitsuite/bunya/0000164708_00001.html

Ministry of Health, New Zealand. 2021. COVID-19: data and statistics. Available at https://www.health.govt.nz/our-work/diseases-and-conditions/covid-19-novelcoronavirus/covid-19-data-and-statistics

Ministry of Health, Singapore. 2021. Updates on COVID-19 local situation. Available at https://www.moh.gov.sg/covid-19

NHK. 2021. New coronavirus: bed usage rate. Available at https://www3.nhk.or.jp/news/special/coronavirus/hospital/

Osaka Prefecture. 2021. Latest infection trends in Osaka Prefecture. Available at https://covid19-osaka.info/

Prime Minister's Office of Japan. 2021. About the new corona vaccine. Available at https://www.kantei.go.jp/jp/headline/kansensho/vaccine.html

R Core Team. 2020. R: a language and environment for statistical computing. Vienna, Austria: $R$ Foundation for Statistical Computing.

Rambaut A, Holmes EC, O'Toole Á, Hill V, McCrone JT, Ruis C, du Plessis L, and Pybus OG. 2020. A dynamic nomenclature proposal for SARS-CoV-2 lineages to assist genomic epidemiology. Nature Microbiology 5:1403-1407. 10.1038/s41564-020-0770-5

Tokyo Metropolitan Government. 2021. Latest infection trends in Tokyo. Available at https://stopcovid19.metro.tokyo.lg.jp/cards/details-of-confirmed-cases/

Tukey JW. 1977. Exploratory data analysis. London: Addison-Wesley Pub. Co.

Wallinga J, and Lipsitch M. 2007. How generation intervals shape the relationship between growth rates and reproductive numbers. Proceedings Biological Sciences 274:599-604. 10.1098/rspb.2006.3754

Wright ES. 2015. DECIPHER: harnessing local sequence context to improve protein multiple sequence alignment. BMC Bioinformatics 16:322. 10.1186/s12859-015-0749-Z

Wu F, Zhao S, Yu B, Chen Y-M, Wang W, Song Z-G, Hu Y, Tao Z-W, Tian J-H, Pei Y-Y, Yuan M-L, Zhang Y-L, Dai F-H, Liu Y, Wang Q-M, Zheng J-J, Xu L, Holmes EC, and Zhang YZ. 2020. A new coronavirus associated with human respiratory disease in China. Nature 579:265-269. 10.1038/s41586-020-2008-3

Yang Z, and Rannala B. 2012. Molecular phylogenetics: principles and practice. Nat Rev Genet 13:303-314. 10.1038/nrg3186

Zhang S-f, Tuo J-I, Huang X-b, Zhu X-b, Zhang D-m, Zhou K, Lei Y, Hong-jiao L, Bo-jian Z, Kwok-yung Y, Meng-feng L, Kai-yuan C, and Lin X. 2018. Epidemiology characteristics of human coronaviruses in patients with respiratory infection symptoms and phylogenetic analysis of HCoV-OC43 during 2010-2015 in Guangzhou. PLOS ONE 13(1): e0191789. https://doi.org/10.1371/journal.pone.0191789

PeerJ reviewing PDF | (2021:03:58872:3:0:NEW 16 Aug 2021) 


\section{Table $\mathbf{1}$ (on next page)}

The domestic variants.

Colours are those indicated in Fig 4A and Fig 3D. The characteristics in PCA used for the definition, peak date, and the mutated amino acids are presented. 


\begin{tabular}{|c|c|c|c|}
\hline Colour & Characteristics & Peak & Residue \\
\hline tangerine & (-) B.1.1.214 & $2020 / 04 / 13$ & $(-)$ \\
\hline water blue & $\begin{array}{l}\text { PC192 >0.00034 } \\
\text { B.1.1.214 }\end{array}$ & 2020/08/03 & $\begin{array}{l}\text { 1a.1361:S/P; 1a.3371:P/S; } \\
\text { N.414:A/V; N.151:P/L }\end{array}$ \\
\hline green & $\begin{array}{l}\text { PC66 }>0.00034 \\
\text { B.1.1.214 }\end{array}$ & 2021/01/04 & $\begin{array}{l}\text { 1b.1567:P/L; 1b.2684:R/l; } \\
\text { N.90:A/T; N.234:M/l; }\end{array}$ \\
\hline blown & $\begin{array}{l}\text { PC114 <-0.00071 } \\
\text { B.1.1.214 }\end{array}$ & 2021/01/04 & $\begin{array}{l}\text { 1b.1567:P/L; 1b.2684:R/l; } \\
\text { N.234:M/I }\end{array}$ \\
\hline purple & $\begin{array}{l}\text { PC78 }>0.0013 \\
\text { B.1.1.214 }\end{array}$ & $2021 / 01 / 04$ & $\begin{array}{l}\text { 1a.110:H/R; 1a.3847:V/I; } \\
\text { 1b.1567:P/L; 1b.2684:R/I; } \\
\text { E.72:L/I; 6.22:F/L; N.234:M/I; }\end{array}$ \\
\hline flesh pink & $\begin{array}{l}\text { new PC5 }<-0.01 \\
\text { B.1.1.215 }\end{array}$ & $2021 / 01 / 10$ & $\begin{array}{l}\text { 1a.727:T/I; 1a.2039:L/F; } \\
\text { 1a.4348:K/R; 1b.1567:P/L; } 1 \\
\text { b.2684:R/I; S.675:Q/H; } \\
\text { N.234:M/I }\end{array}$ \\
\hline orange & $\begin{array}{l}\text { PC171 > } 0.00088 \\
\text { B.1.1.214 }\end{array}$ & $2021 / 01 / 11$ & $\begin{array}{l}\text { 1a.2702:Q/H; 1a.2981:S/F; } \\
\text { 1b.1567:P/L; 1b.2684:R/I; } \\
\text { S.720:I/V; N.234:M/I; }\end{array}$ \\
\hline coral red & $\begin{array}{l}\text { new PC15 }<-0.009 \\
\text { PC66 }<0.00034 \\
\text { B.1.1.214 }\end{array}$ & $2021 / 01 / 16$ & $\begin{array}{l}\text { 1a.2259:M/I; 1a.3722:Y/C; } \\
\text { 1b.1567:P/L; 1b.2143:A/S; } \\
\text { 1b.2684:R/I; S.261:G/V; } \\
\text { S.675:O/H; N.234:M/I }\end{array}$ \\
\hline blue green & $\begin{array}{l}\mathrm{PC} 30<-.002 \\
\text { R.1 }\end{array}$ & $2021 / 03 / 29$ & $\begin{array}{l}\text { 1b.1362:G/R; 1b.1936:P/H; } \\
\text { S.152:W/L; S.484:E/K; S.769:G/V; } \\
\text { M.28:F/L; N.1-101:M/-; } \\
\text { N.187:S/L; N.418:Q/H }\end{array}$ \\
\hline
\end{tabular}

2 Table 1 The domestic variants. Colours are those indicated in Fig 4A and Fig 3D. The characteristics in 3 PCA used for the definition, peak date, and the mutated amino acids are presented. 


\section{Figure 1}

Changes in groups of variants found in Japan.

(A) The scaled principal component (sPC) of the sequence found in Japan. Axes PC1 and PC2. Four groups are obvious: the clearly separated groups common to other countries (Konishi 2021c). Numbers from 0 to 3 are tentatively assigned. (B) Increase/decrease of each group. Group 3 was dominant until March 2020; Group 1 then became dominant, and Group 0 was rapidly increasing in May 2021, reflecting the increase of B.1.160.1 (left axis). The green background indicates the periods of the states of emergency, and the pink background reflects the Go To travel campaign period, which is currently suspended. The thick blue line is the number of confirmed cases, and the thin blue line is $1 / 20$ the number of PCR tests (right axis). PCR is performed only on symptomatic patients, and the total number of tests was, on average, 19 times the number of positives. The grey dotted line is the number of arriving visitors.
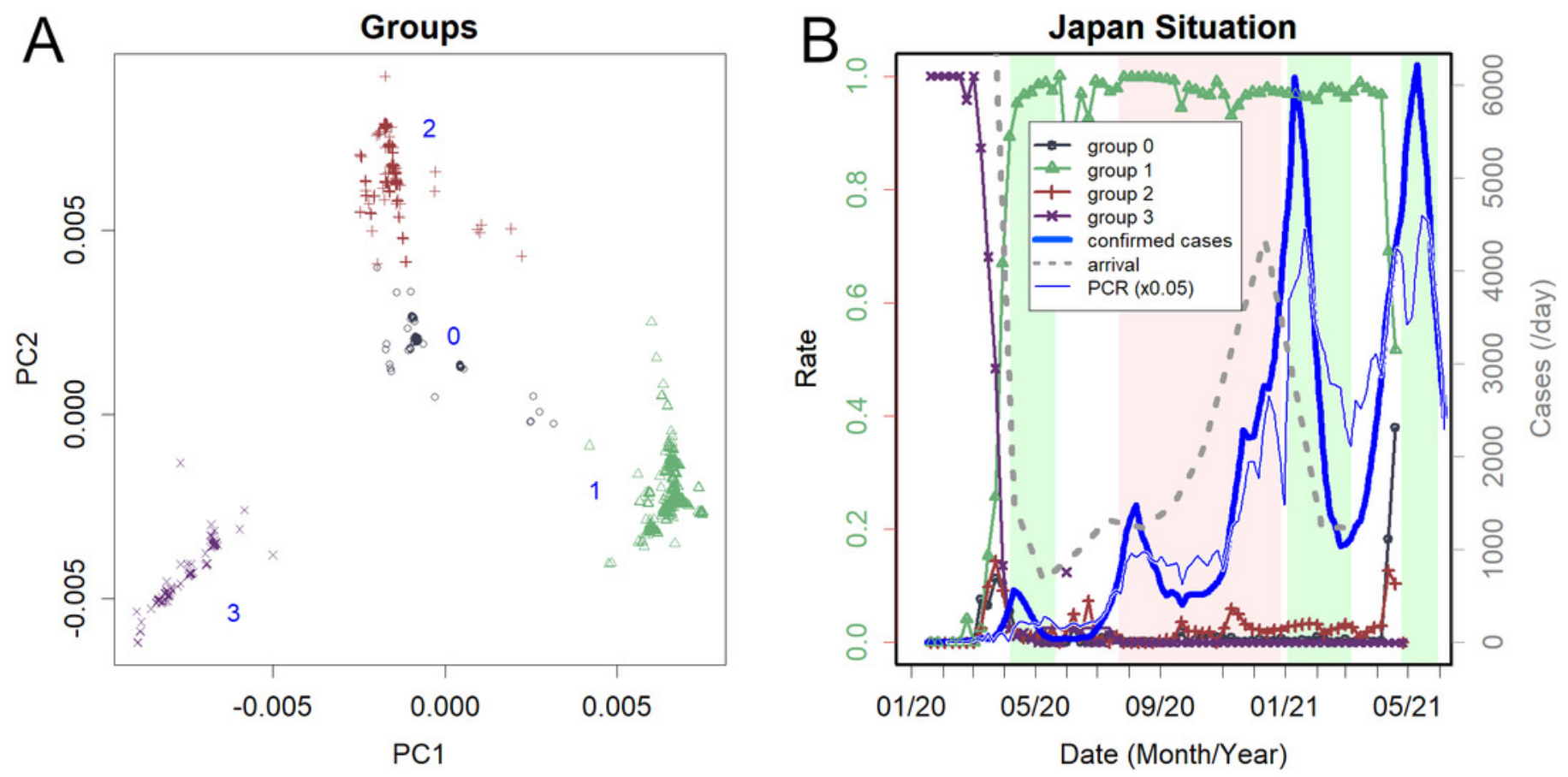


\section{Figure 2}

Examples of some variants, time of detection, and PC values.

The colors are the same as in Fig. 4A. (A) The variant that caused the second peak. (B) The newest prevalent variant, R.1, among the domestic variants. (C) The variant that was dominant in the third peak and that also spread to South America. (D) Variant B.1.1.7, the so-called England new variant. The PC values are substantially different due to many mutations. 

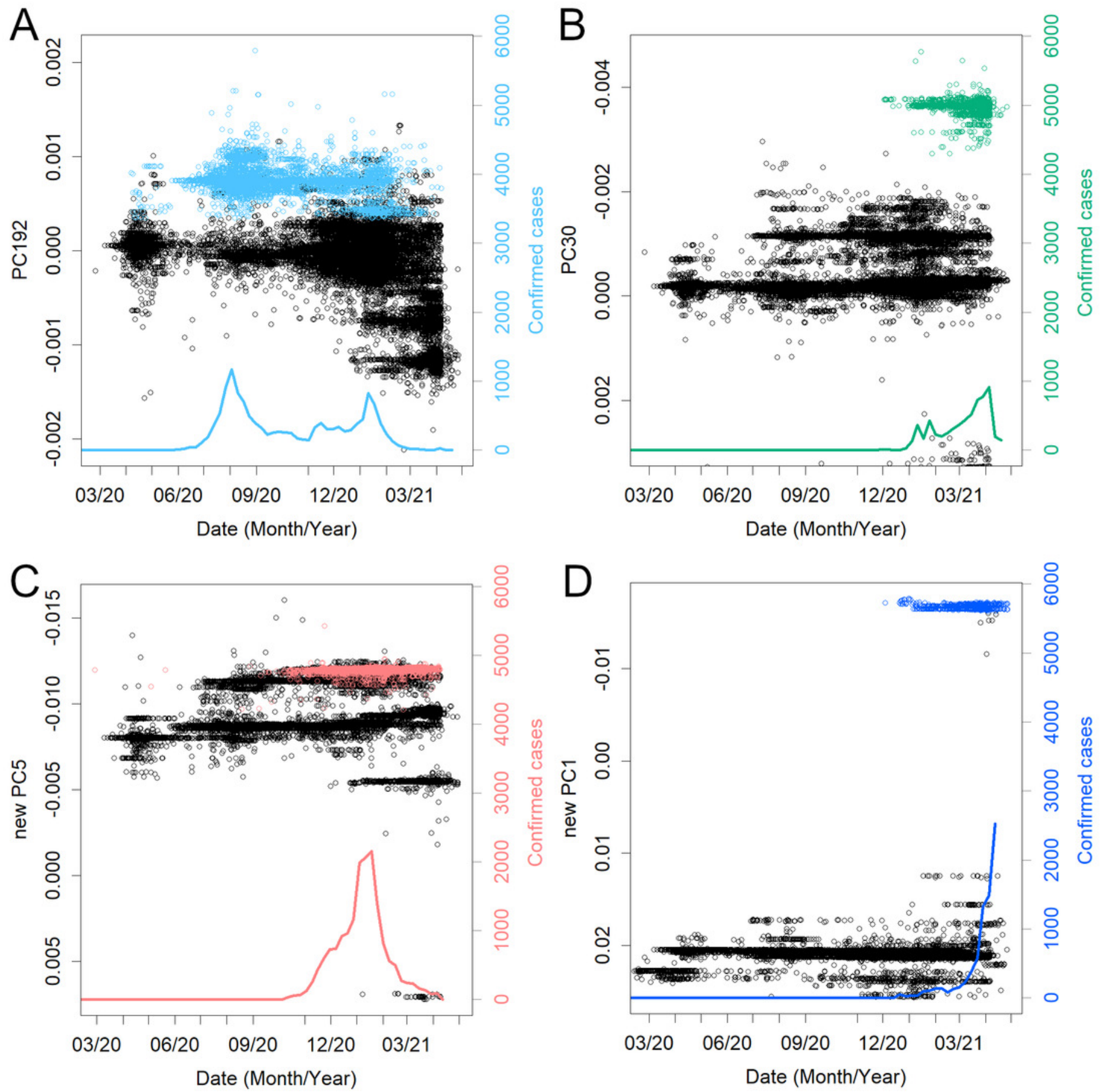


\section{Figure 3}

Details of the number of sequences reported.

(A) Number of sequences for groups 1, 2, and 0 in November 2020, a semi-log plot. Group 1 is overwhelmingly large. Variants from overseas appeared. Variant B.1.2 was predominant in the USA. (B) December 2020. Variant B.1.1.7 appeared. Variant B. 177 swept Europe. (C) January 2021. Variant B.1.160 was predominant in France. (D) Changes in the rate of the whole in 2021. Variant B.1.617.1 was predominant in India. 


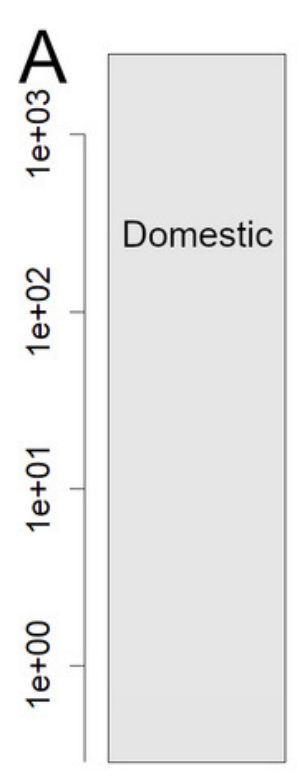

Group.1

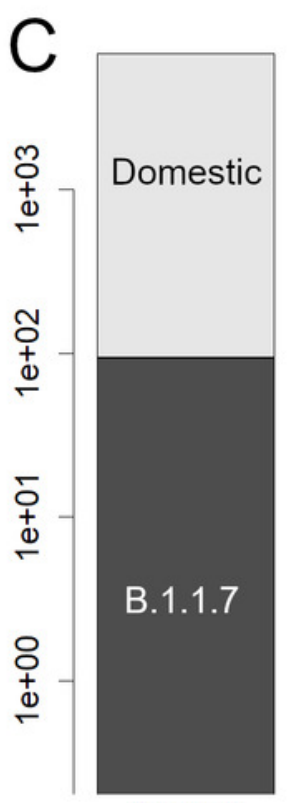

Group.1
Nov 2020

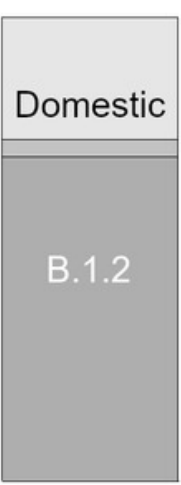

Group.2

Jan 2021

Domestic

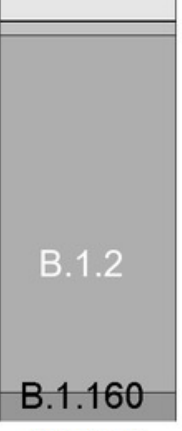

Group.2

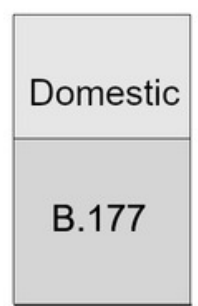

Group.0

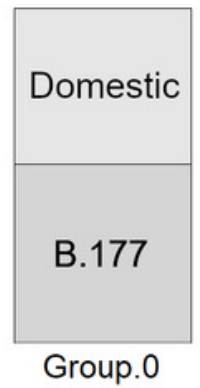

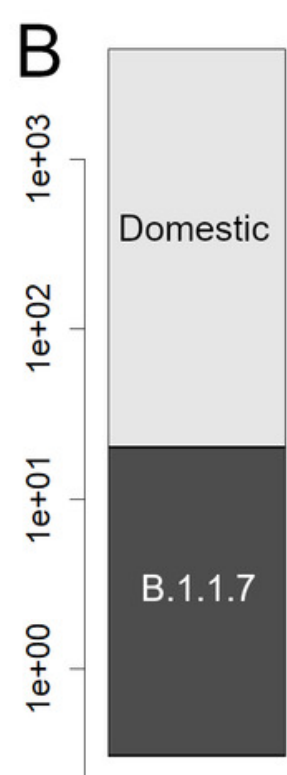

Group.1
Dec 2020

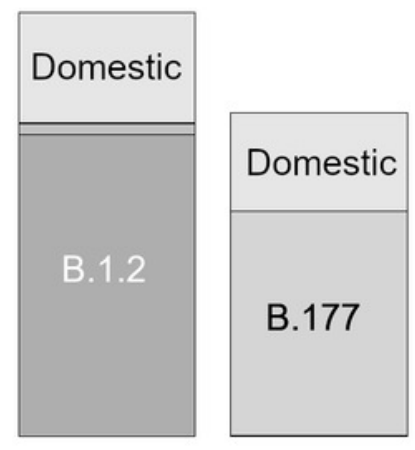

Group.2 Group.0

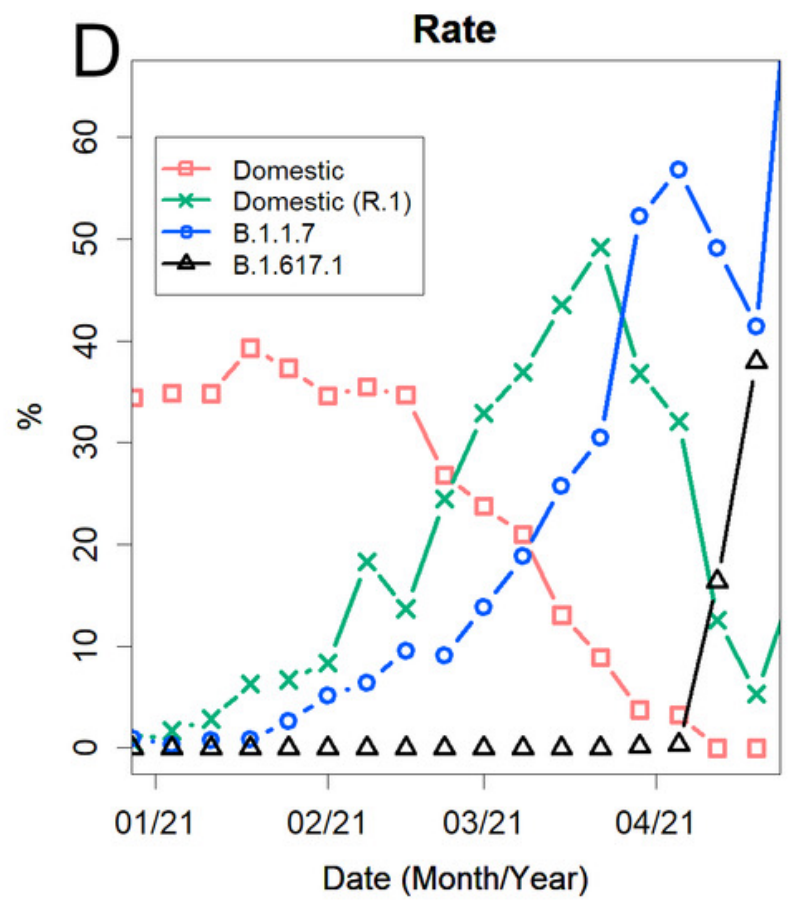




\section{Figure 4}

Changes in the number of infected people.

(A) Logarithmic growth rate (black) estimated from the total confirmed cases. The dashed lines in light blue and orange are the approximated straight lines, and the numbers are their slopes. The decreased rates of $K$ were almost always the same. The black dotted line is the $K$ of variant B.1.1.7. The thick gray line is the number of overall cases. Colored lines are domestic variants in group 1 (Table 1). The increasing blue line is B.1.1.7. The black dashed line increasing at the end of the panel is B.1.617.1. The green background indicates the state of emergency, and the pink background reflects the period of the Go To travel campaign. (B) Comparison between the number of cases nationwide and the number of cases in Tokyo alone. The blue line is $K$ in Tokyo. (C) Estimated basic reproduction number (black), which has also changed because of the change in $K$. 

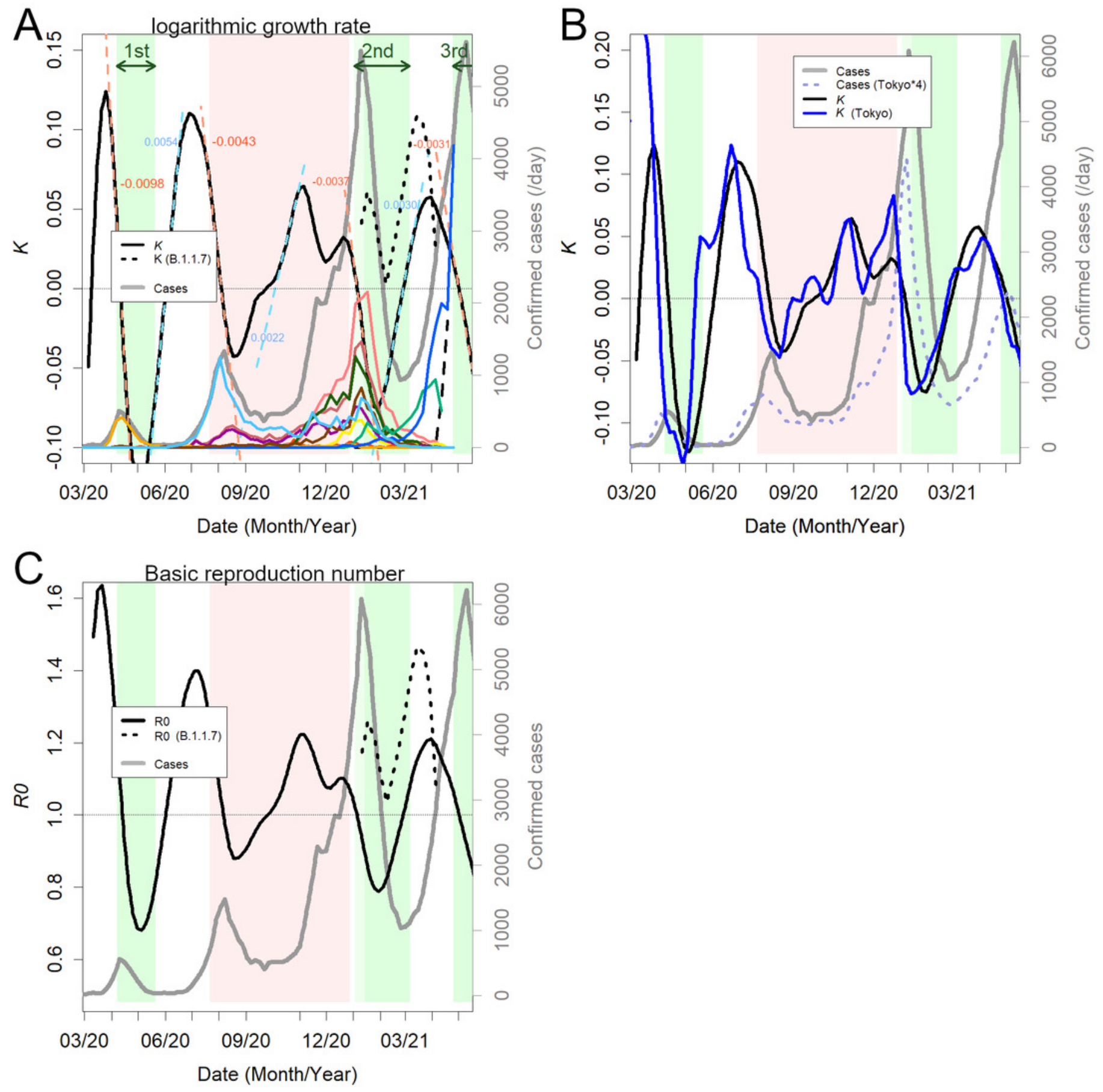
Figure 5

Changes observed in variant B.1.1.7.

(A) The first green variant changed gradually to blue and then brown with three amino acid changes each. The dots represent SPC20 values, and the lines represent the rate of each among all B.1.1.7. (B) sPC11. One amino acid mutation from blue produced coral-red, and this difference was detected on this axis. The lines show the change in $K$. After the growth of the initial variant was suppressed, the mutated variants created the source of the next peak.
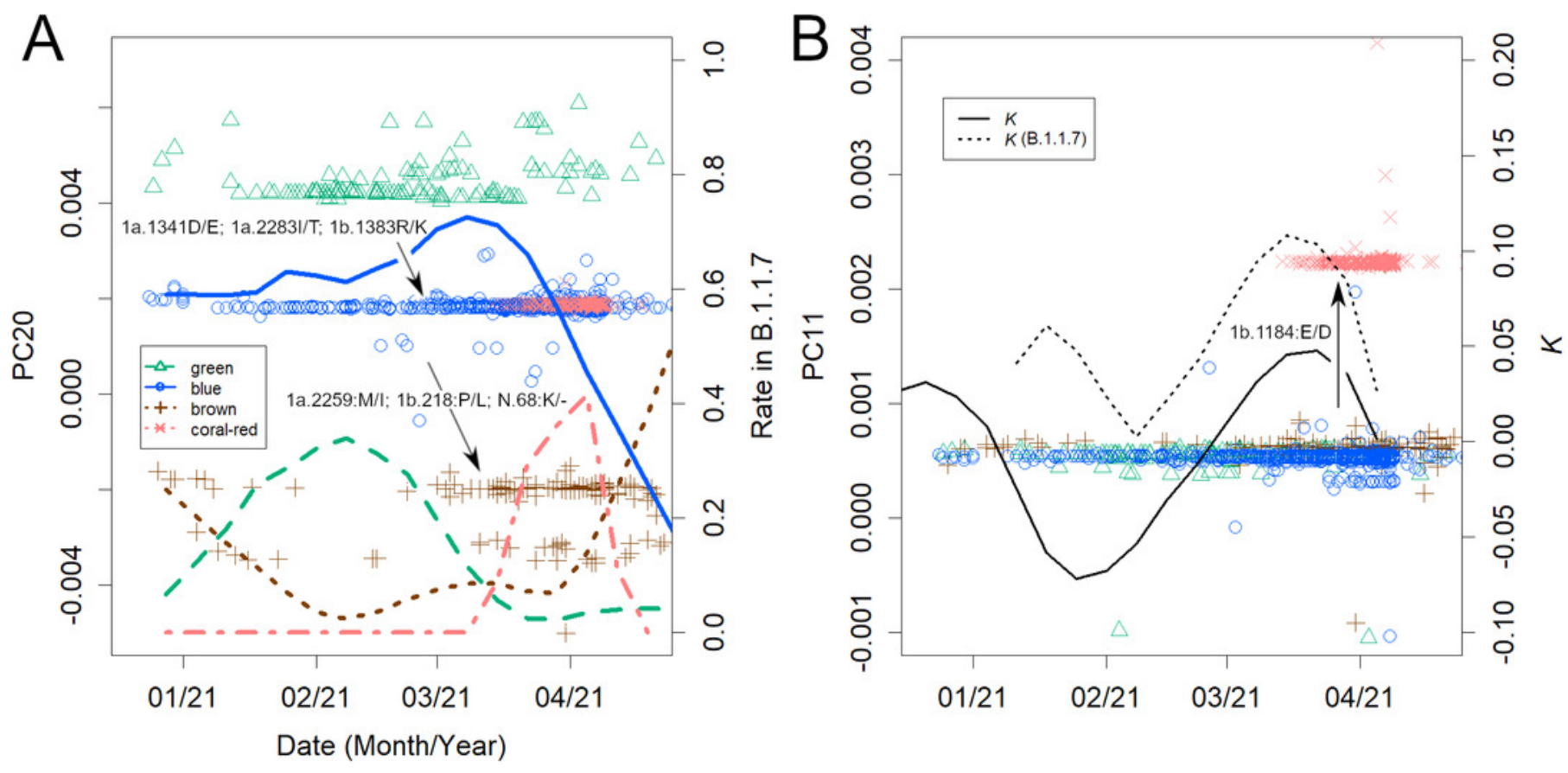